\title{
Postpartum Ovarian Vein Thrombosis Associated with Lower Uri- nary Tract Infection and Vaginitis
}

\author{
Shannon Tomita ${ }^{\text {** }}$ \\ ${ }^{1}$ Department of Obstetrics and Gynecology, Icahn School of Medicine at Mount Sinai, New York NY
}

"Corresponding author: Shannon Tomita, Department of Obstetrics and Gynecology, Icahn School of Medicine at Mount Sinai, New York NY, Tel: + 310 408-2380; E-mail: satomita@gmail.com

Citation: Tomita S (2017) Postpartum Ovarian Vein Thrombosis Associated with Lower Urinary Tract Infection and Vaginitis. Gynecol Obstet Open Acc OBOA, 2017:102. DOI: 10.29011/ OBOA-102.100002

Received Date: 9 March, 2017; Accepted Date: 9 May, 2017; Published Date: 17 May, 2017

\section{Background}

Ovarian Vein Thrombosis (OVT) is a rare event, usually provoked by infection in the postpartum period. The presentation can mimic many other disease processes and a high index of suspicion is necessary for correct diagnosis, which is best made by CT. The most serious sequelae include sepsis and pulmonary embolism. Patients with OVT are frequently found to have an underlying thrombophilia.

Case: 39 year old G11P2092 post-op day 21 from a repeat cesarean delivery and bilateral tubal ligation presenting with acute onset left lower quadrant pain is found to have extensive left ovarian vein thrombosis.

Conclusion: We present an unusual case of a postpartum patient who presented with left sided OVT, possibly precipitated by lower urinary tract infection and/or vaginitis.

\section{Teaching Points}

- Postpartum ovarian vein thrombosis can present without fever or an obvious source of infection and a high clinical index of suspicion must be maintained to avoid morbidity associated with potential sequelae of untreated thrombosis

- Once diagnosis is made, thorough and detailed patient counseling regarding the potential life-threatening consequences of interrupted or unfinished treatment is imperative for optimal patient compliance with outpatient anticoagulation

\section{Introduction}

Postpartum Ovarian Vein Thrombosis (OVT) is extremely rare. The clinical presentation can mimic many other disease processes and a high index of suspicion is necessary to obtain the correct imaging for diagnosis. The majority of patients with postpartum OVT presents with signs and symptoms of infection and a right-sided thrombus. These patients are also frequently found to have an underlying thrombophilia, which predisposes them to recurrence or other venous thromboembolic events if anticoagulation treatment is delayed or interrupted.

Case: A 39-year-old female G11P2092 presented to the emergency room 22 days after a repeat cesarean section with bilateral tubal ligation performed at an outside hospital for acute onset of left lower quadrant pain. The pain was described as intermittent, stabbing in quality, radiating to her lower back, and 10 out of 10 in severity on the pain scale. She denied any alleviating or aggravating factors. Review of systems was otherwise negative, with pertinent negatives including fever, chills, dysuria, diarrhea or constipation, nausea, vomiting, abnormal vaginal discharge. Her antepartum and intrapartum course were uncomplicated. She denied personal or family history of blood clots or known clotting disorders. Upon evaluation, the patient was afebrile and appeared stable. Her vital signs were within normal limits. Physical exam was only significant for moderate tenderness in the left adnexa, without peritoneal signs. She had no CVA tenderness. No masses or fundal tenderness was appreciated on bimanual exam and no abnormal vaginal discharge or bleeding on speculum exam.

CBC was significant only for mild leukocytosis $(11,800)$ with no left shift. Basic metabolic panel and hepatic function panel were within normal limits. Her urinalysis was positive for moderate leukocytes esterase and 25-50 WBC, with a urine culture that eventually grew $>100,000 \mathrm{cfu} / \mathrm{mL}$ E. coli. A vaginal swab also returned positive for bacterial vaginosis. CT abdomen and pelvis with oral and intravenous contrast revealed extensive dilatation and thrombus within the entire left gonadal vein associated with inflammatory changes extending to the left ureter with perinephric stranding. These findings were consistent with left gonadal vein thrombophlebitis and possible pylitis.

The patient was admitted to the gynecology service and began treatment with anticoagulation therapy and IV antibiotics. She received twice daily, weight-based dosing of low molecular weight heparin $(1 \mathrm{mg} / \mathrm{kg})$ and Unasyn $3 \mathrm{~g}$ daily. Vascular surgery 


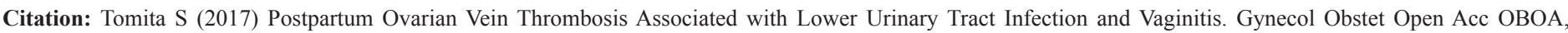
2017:102.

was consulted and agreed with management plan. Once her urine culture was resulted, her antibiotics were switched to ceftriaxone in accordance with reported sensitivities.

Hospital course was uncomplicated. A repeat CT scan on hospital day 4 showed a decrease in the caliber of the vein as well as a decrease in surrounding inflammatory changes. A thrombophilia work-up was only significant for decreased protein $\mathrm{S}$, which is normal for postpartum period. She was discharged home on hospital day 4 with instructions to continue anticoagulation for three months and follow-up with vascular surgery.

Comment: The highest reported incidence of ovarian vein thrombosis is $0.18 \%$ following vaginal delivery, and $1-2 \%$ following cesarean delivery [1]. Most of the information known about ovarian vein thrombosis is thus derived from case reports and a few retrospective studies.

Over $80 \%$ of OVT cases occur postpartum, when women have multiple known risk factors for thrombus formation [2]. Pregnant women remain in a hypercoagulable state for 6-12 weeks after delivery due to increased levels of coagulation factors, increased platelet adhesion, decreased fibrinolysis and decreased protein S levels $[3,5]$. Venous stasis results from compression of pelvic vasculature by the gravid uterus as well as an increased diameter of the ovarian venous system due to increased blood flow and progesterone influence [4]. In addition, in vaginal or cesarean delivery, the pelvic vasculature undergoes a degree of endothelial damage [3]. All 3 components of Virchow's Triad are thereby satisfied.

Sixty percent of patients with postpartum OVT will present with signs or symptoms of infection [2]. Bacterial injury to the venous endothelium has been suggested as an initiating step in the formation of ovarian venous thrombosis as a result of any abdominal or pelvic infectious process [4]. Reported sources of infection associated with OVT include endometritis, chorioamnionitis, lower urinary tract infection, vaginitis, extensive wound infection, pelvic inflammatory disease, inflammatory bowel disease, appendicitis, and clostridium difficile associated diarrhea [4]. The extensive communication between various pelvic venous plexuses provides adequate means of bacterial seeding into the ovarian vasculature with any of these infectious sources [4]. Other known risk factors aside from acute infection include malignancy, non-surgical trauma, thrombophilia and pelvic surgery [2]. With pelvic surgery as a known risk factor, it seems plausible that bilateral tubal ligation, even if it occurs during cesarean section, could potentially be an independent risk factor for the development of OVT given the possibility of disrupting the venous plexus in the broad ligament or introducing bacteria during the procedure. In the existing retrospective reviews, tubal ligation is not addressed or explored as a risk factor in patients with postpartum OVT. True idiopathic cases of ovarian vein thrombosis are exceedingly rare, with less than 10 reported cases published.
Most patients present within the first 4 weeks after delivery with acute abdominal pain with associated abdominal tenderness on exam [6]. Postpartum patients will also commonly present with a fever and leukocytosis (60\%) [2]. The clinical features of non-postpartum OVT is much more variable and nonspecific, especially in the setting of malignancy wherein it is often an incidental finding.

The ovarian vein is the most commonly involved vessel in postpartum pelvic thrombophlebitis [5]. The majority of cases are right-sided followed by bilateral involvement, with the least number of cases reported as left-sided [6]. Dextrorotation of the gravid uterus in pregnancy is thought to increase venous stasis on the right side [6]. Additionally, the right ovarian vein is thought to be longer, more tortuous and to have more incompetent valves and more anterograde flow than its counterpart on the left side [6].

Diagnosis can be made by ultrasound, MRI or CT scan with sensitivities of $52 \%, 92 \%$ and $100 \%$ respectively [1]. CT is particularly useful for assessing the extent of thrombosis within the ovarian vein and evaluating for involvement of surrounding vasculature, more specifically the renal vein and the IVC [1]. Assessment of the superior extension of the thrombus may be difficult on CT due to a mixing effect of the contrast medium at the level of the renal veins [1]. If the superior extension of the thrombus must be accurately visualized, MRI could potentially provide more information [1]. Concurrent, non-fatal pulmonary embolism was diagnosed in just under $10 \%$ of patients, and other related thrombotic events such as splenic infarction were found in even fewer patients.

Treatment includes anticoagulation with twice daily dosing of weight based low molecular weight heparin as well as IV antibiotics if the patient presented with signs of infection or an obvious source of infection. One multicenter study followed 24 patients after discharge for resolution of thrombus and found that approximately $65 \%$ of ovarian veins will completely recanalize, $15 \%$ will partially recanalize and $20 \%$ will demonstrate persistent occlusion on follow-up imaging [3]. Another multicenter review conducted over 15 years with 74 patients included, however, demonstrated complete resolution of thrombus in all patients at approximately 5 month follow-up on CT and/or ultrasound with Doppler imaging [2]. In the same study, all patients had a thrombophilia workup on admission. Twenty percent of patients with pregnancy related ovarian vein thrombosis and $42.9 \%$ of patients with non-pregnancy related ovarian vein thrombosis tested positive, suggesting that every patient who presents with ovarian vein thrombosis should undergo a thrombophilia workup to prevent recurrence or other thromboembolic events [2]. There is currently no standardized treatment protocol, however most patients are treated with anticoagulation for an average of 3 months. Patients with pregnancy/ postpartum related OVT tended to be treated longer than 3 months and also tended to need outpatient antibiotic treatment [2]. 


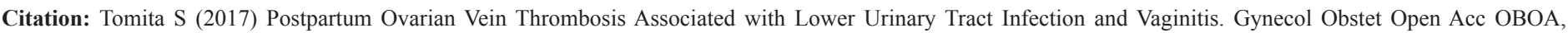
2017:102.

The sequelae of ovarian venous thrombosis include pelvic congestion syndrome, sepsis and pulmonary embolism [3,5]. In the reported cases, sepsis usually resulted from delayed diagnosis wherein the patient had been admitted for treatment of endometritis or fever of unknown origin and found to repeatedly fail antibiotic treatment before correct diagnosis was made [2]. Pulmonary embolism, when not diagnosed concurrently, was typically diagnosed in patients in whom anticoagulation therapy was delayed, interrupted or stopped prematurely [2]. Mortality from pulmonary embolism has only been observed in patients with malignancy associated OVT [3].

In conclusion we present an unusual case of a postpartum patient presenting with a left-sided ovarian vein thrombus likely precipitated by lower urinary tract infection and possibly vaginitis. A high clinical index of suspicion and expeditious diagnosis are necessary to avoid the more serious sequelae of OVT and extensive patient counseling is imperative to ensure appropriate compliance with treatment and follow-up.

\section{Reference}

1. Sharma P, Abdi S (2012) Ovarian Vein Thrombosis. Clinical Radiology 25: 893-898.
2. Rottenstreich A, Da'as N, Kleinstern G, Spectre G, Amsalem H. (2016) Pregnancy and non-pregnancy related ovarian vein thrombosis: Clinical course and outcome. Thrombosis Research 146: 84-88.

3. Labropoulos N, Malgor R, Comito M, Gasparis A, Pappas P, et al. (2014) The natural history and treatment outcomes of symptomatic ovarian vein thrombosis. Journal of Vascular Surgery and Venous and Lymphatic Disorders. July 15; 3: 42-47.

4. Heavrin B, Wrenn K (2007)Ovarian vein thrombosis: a rare cause of abdominal pain outside the peripartum period. Journal of Emergency Medicine 34: 67-69.

5. Jenayah AA, Saoudi S, Boudaya Fethia, Bouriel I, Sfar Ezzeddine, et al. ( 2015) Case report: Ovarian vein thrombosis. Pan African Medical Journal.21: 251

6. Tait C, Baglin T, Watson H, Laffan M, Makris M, et al. (2012) Guidelines on the investigation and management of venous thrombosis at unusual sites. British Journal of Hematology159: 28-38.

7. Wysokinska EM, Hodge D, McBane RD (2006) Ovarian vein thrombosis: Incidence of recurrent venous thromboembolism and survival. 